\title{
Lesson of the month 2: Catecholamine-induced cardiomyopathy - pitfalls in diagnosis and medical management
}

\author{
Authors: Yaasir Mamoojee, ${ }^{A}$ Munawar Arham, ${ }^{B}$ Wael Elsaify ${ }^{C}$ and Sath $\mathrm{Nag}^{\mathrm{D}}$
}

Cardiomyopathy as the initial presentation of phaeochromocytoma (PCA) is uncommon. Diagnostic work-up and perioperative management may be challenging within this context. We report three cases of PCA presenting with cardiomyopathy to illustrate the pitfalls in diagnosis and management. None of the patients had typical adrenergic symptoms and all three were established on beta-blockers prior to diagnosis. Their fractionated plasma catecholamine levels were elevated and the diagnosis of PCA was confirmed with various imaging modalities and post adrenalectomy. Interpretation of fractionated catecholamine levels in the context of established cardiomyopathy is difficult as cardiac failure of any aetiology generates an adrenergic response. Hence screening all patients with idiopathic cardiomyopathy is likely to generate a high false-positive rate. However, a high index of suspicion should prompt further diagnostic work-up in patients with idiopathic cardiomyopathy for occult PCAs. Peer-reviewed guidelines are required to guide the investigation and management of suspected catecholamine-induced cardiomyopathy.

KEYWORDS: Phaeochromocytoma, cardiomyopathy, catecholamine, diagnosis

\section{Background}

The estimated annual incidence of phaeochromocytoma (PCA) is approximately 0.8 per 100,000 person years. ${ }^{1}$ Classical clinical presentations with adrenergic symptoms include episodic headaches, tachycardia, sweating, and paroxysmal or sustained hypertension. ${ }^{2}$ Cardiomyopathy is however far less common as the index presentation. The diagnostic investigation for PCA in patients with cardiomyopathy is problematic. Biochemical

Authors: ${ }^{\mathrm{A} S p R}$ in diabetes and endocrinology, The James Cook

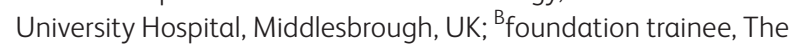
James Cook University Hospital, Middlesbrough, UK; ${ }^{C}$ consultant in general and endocrine surgery, The James Cook University Hospital, Middlesbrough, UK; ${ }^{D}$ consultant in diabetes and endocrinology, The James Cook University Hospital, Middlesbrough, UK and radiological investigations have variable performance characteristics. ${ }^{3-5}$ Once diagnosed the medical management can be further challenging. We report three cases of PCA presenting with cardiomyopathy to illustrate the pitfalls in diagnosis and management. None of the patients had typical adrenergic symptoms and all of them were well established on a beta-blocker prior to diagnosis. A PCA was confirmed on histology post-adrenalectomy.

\section{Case reports}

\section{Patient 1}

A 66-year-old male was referred with an incidental 3.1-cm heterogeneous right adrenal mass with an attenuation of 56 Hounsfield units (HU) on computerised tomography (CT) imaging. He had a past medical history of atrial fibrillation, severe left ventricular (LV) impairment and non-flow limiting coronary disease. His regular medications included bisoprolol, ramipril, digoxin, furosemide and warfarin. His plasma and 24-h urinary metadrenaline and normetadrenaline levels were raised (Figs 1 and 2). A metaiodobenzylguanidine (MIBG) scan showed avid uptake at the level of the right adrenal gland which corresponded to the area seen on the CT (Fig 3). Alpha blockade prior to surgery was complicated with significant postural hypotension on only $10 \mathrm{mg}$ phenoxybenzamine.

\section{Patient 2}

A normotensive 56-year-old male with dilated cardiomyopathy and atrial fibrillation was found to have an incidental longstanding $3-\mathrm{cm}$ right adrenal nodule with an attenuation of $48 \mathrm{HU}$ on CT. His regular medications included bisoprolol, losartan, eplerenone and warfarin. His plasma and urine metadrenaline levels were raised during decompensated heart failure and improved with optimisation of heart failure treatment (Figs 1 and 2). However, a paradoxical increase in plasma catecholamine levels was noted despite clinical and echocardiographic improvement in cardiac function. MIBG and octreotide scans were both negative but CT imaging of the right adrenal lesion with wash-out studies showed indeterminate characteristics. He only tolerated phenoxybenzamine at $10 \mathrm{mg}$ twice daily prior to surgery due to postural hypotension. 


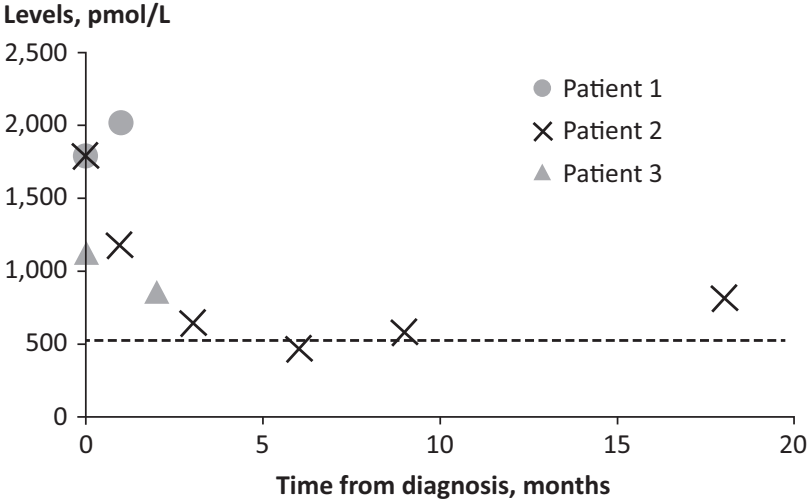

Fig 1. Plasma metadrenaline levels prior to surgery (reference value 80-510 pmol/L).

\section{Patient 3}

A 61-year-old female with neurofibromatosis type-1 developed acute heart failure while on holiday in Greece. This was complicated by acute left lower limb ischaemia secondary to embolisation from a $\mathrm{LV}$ thrombus. On return to the UK her repeat echocardiography revealed normal LV function and absence of LV thrombus. A diagnosis of takotsubo cardiomyopathy was then postulated. She was weaned off all cardiac medications including carvedilol. Her fractionated metadrenaline levels remained elevated and a subsequent 2.4-cm left adrenal mass with an attenuation of $43 \mathrm{HU}$ was found on abdominal CT scan. This corresponded to an increased uptake in the left adrenal on an MIBG scan. She tolerated phenoxybenzamine at $100 \mathrm{mg}$ daily. A beta-blocker was later reinstituted prior to surgery.

\section{Literature review}

We found 74 cases of PCA presenting primarily with cardiac crises between 2000 and 2014 in English databases (see online-only supplementary material: S1). Table 1 shows the demographic and presenting characteristics of these patients. The median age at presentation was 41 years with

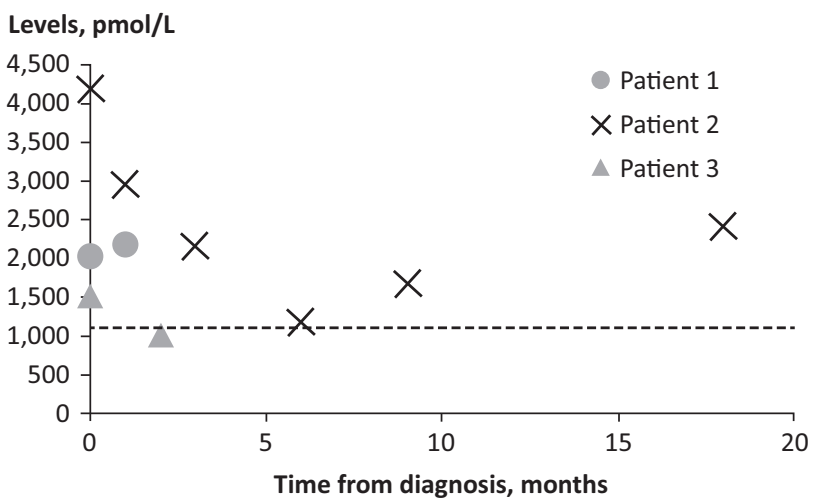

Fig 2. Plasma normetadrenaline levels prior to surgery (reference value $120-1,180 \mathrm{pmol} / \mathrm{L})$.
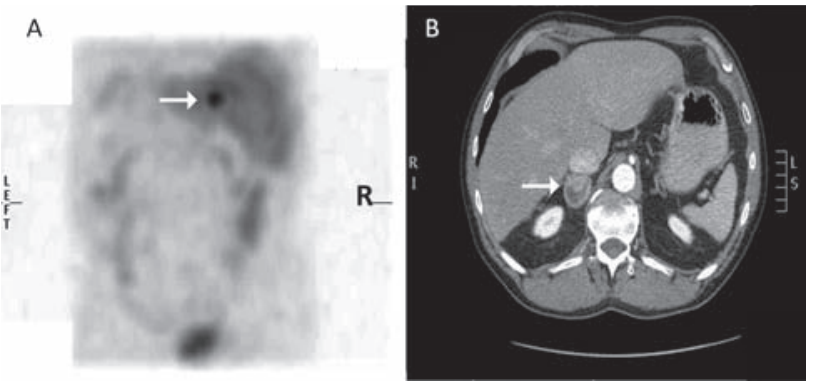

Fig 3. MIBG-avid right adrenal uptake (white arrow in A) corresponding to a heterogenous right adrenal mass on $\mathrm{CT}$ (white arrow in $\mathrm{B}$ ).

a female preponderance of $59 \%$. The presence of adrenergic symptoms was only documented in 27 cases $(36 \%)$.

Although the majority of patients were hypertensive (63\%), a sizeable minority was normotensive (11\%). The incidence of takotsubo cardiomyopathy was relatively overrepresented in this population at $28 \%$ compared to $2.2 \%$ in patients presenting with acute coronary syndromes in the literature. ${ }^{6}$ All adrenal lesions were over $2 \mathrm{~cm}$ in size with almost equal distribution between adrenal sides.

Table 1. Characteristics of 74 cases of PCA crises

presenting with cardiomyopathy between 2000 and 2014 in the literature.

\begin{tabular}{ll} 
Parameter & Value \\
Median age, years (range) & $41(13-72)$ \\
Female gender, $\mathrm{n}(\%)$ & $44 / 74(59)$ \\
Prior adrenergic symptoms, $\mathrm{n}(\%)$ & $27 / 74(36)$ \\
BP at presentation, $\mathrm{n}$ (\%) & $63 / 74$ \\
Hypertension & $40(63)$ \\
Labile BP & $11(17)$ \\
Hypotension & $5(8)$ \\
Normotension & $7(11)$ \\
Type of cardiomyopathy, $\mathrm{n}$ (\%) & $74 / 74$ \\
Takotsubo & $21(28)$ \\
Myocarditis & $3(4)$ \\
LVSD & $40(54)$ \\
Unspecified & $10(14)$ \\
PCA site, $n$ ( \%) & $58 / 74$ \\
Right adrenal & $23(40)$ \\
Left adrenal & $25(43)$ \\
Bilateral & $4(7)$ \\
Extra-adrenal & $6(10)$ \\
Mean size of adrenal PCA, cm (range) & $5.8(2.1-14)$ \\
\hline BP = blood pressure; LVSD = left ventricular systolic dysfunction; & PCA $=$ \\
phaeochromocytoma. &
\end{tabular}




\section{Discussion}

Screening for PCA in patients with cardiomyopathy is problematic as cardiac failure of any aetiology generates an adrenergic response ${ }^{7}$ hence interpretation of biochemical tests for catecholamine excess is difficult. The routinely used screening tests (catecholamine profiles) have not been validated in patients with cardiac failure. ${ }^{3}$

Screening all patients with idiopathic cardiomyopathy for catecholamine excess is highly likely to generate false-positive results, and is therefore not a recommended strategy. In addition the incidence of adrenal incidentalomas is rising due to the increasing use of abdominal CT imaging studies for other diagnostic purposes. With most of these CT scans being performed in the older patient group, a significant proportion of these incidental adrenal lesions is expected to coexist in patients with cardiac failure of known or unknown aetiology. Current endocrine guidelines on the investigation of adrenal incidentalomas in this subpopulation are less clear. ${ }^{8}$

A high index of suspicion of occult PCA in patients with idiopathic cardiomyopathy or with an adrenal incidentaloma and cardiac failure should prompt further diagnostic workup. Factors contributing to a high pre-test probability of PCA are: younger age, genetic predisposition to PCA (eg neurofibromatosis type 1 , multiple endocrine neoplasia, von Hippel-Lindau syndrome), presence of adrenergic symptoms, blood pressure anomalies (oscillating blood pressure, hyper and/ or hypotension), takotsubo cardiomyopathy and radiological characteristics of PCA. ${ }^{2,4}$ The latter includes adrenal lesions of heterogeneous composition, increased attenuation $(>20 \mathrm{HU})$, delayed contrast medium washout and possibly $>2 \mathrm{~cm}$ in size $\mathrm{e}^{5}$. The role of CT imaging with washout studies as second-line diagnostic imaging modality in this population requires special consideration given that one of our patients had negative MIBG and octreotide scans but the washout studies were the only abnormal investigations revealing indeterminate characteristics. Once diagnosed the medical management of PCA prior to surgery may be difficult in the context of heart failure. Although beta-blockade without prior alpha-blockade is well known to provoke hypertensive PCA crises, this was not the case in our patients. ${ }^{9}$ After a multidisciplinary consultation it was felt that stopping a well-tolerated beta-blocker prior to alpha-blockade may increase the risk of cardiac decompensation. However management of blood pressure during phenoxybenzamine titration on beta-blockers proved tricky.

\section{Conclusions}

Investigating patients with established cardiomyopathy for possible PCA remains challenging. Routine screening tests are difficult to interpret. A high index of suspicion should prompt further diagnostic work-up with judicious use of currently available imaging modalities as a second-line investigative strategy. Peer-reviewed investigative guidelines are needed for this subgroup of patients.

\section{References}

1 Beard CM, Sheps SG, Kurland LT et al. Occurrence of pheochromocytoma in Rochester, Minnesota, 1950 through 1979. Mayo Clin Proc 1983;58:802-4.

2 Pacak K, Linehan WM, Eisenhofer G et al. Recent advances in genetics, diagnosis, localization, and treatment of pheochromocytoma. Ann Intern Med 2001;134:315-29.

3 Lenders JW, Pacak K, Walter M et al. Biochemical diagnosis of pheochromocytoma: which test is best. JAMA 2002;287:1427-34.

4 Guerrero MA, Schreinemakers JM, Vriens et al. Clinical spectrum of pheochromocytoma. J Am Coll Surg 2009;209:727-32.

5 Hamrahian AH, Ioachimescu AG, Remer EM et al. Clinical utility of noncontrast computed tomography attenuation value (hounsfield units) to differentiate adrenal adenomas/hyperplasias from nonadenomas: Cleveland Clinic experience. J Clin Endocrinol Metab 2005;90:871-7.

6 Kurowski V, Kaiser A, Von Hof K et al. Apical and mid ventricular transient left ventricular dysfunction syndrome (tako-tsubo cardio-myopathy): frequency, mechanisms, and prognosis. Chest 2007;132:809-16.

7 Francis GS, Goldsmith SR, Levine TB, Olivari MT, Cohn JN. The neurohumoral axis in congestive heart failure. Ann Intern Med 1984;101:370-7.

8 Lenders JW, Duh QY, Eisenhofer G et al. Pheochromocytoma and paraganglioma: an endocrine society clinical practice guideline. J Clin Endocrinol Metab 2014;99:1915-42.

9 Sibal L, Jovanovic A, Agarwal SC, Peaston RT et al. Phaeochromocytomas presenting as acute crises after beta blockade therapy. Clin Endocrinol (Oxf) 2006;65:186-90.

Address for correspondence: Dr Y Mamoojee, Department of Diabetes and Endocrinology, The James Cook University Hospital, Middlesbrough TS4 3BW, UK.

Email:ymamoojee@nhs.net 